\title{
Vulnerabilidad sísmica del sistema estructural prefabricado gran panel Soviético en edificios deteriorados y transformados
}

\section{Seismic vulnerability of the prefabricated Soviet great panel system in deteriorated and transformed buildings}

\section{Yamila C. Socarrás ${ }^{1}$ y Eduardo R. Álvarez ${ }^{2}$}

${ }^{1}$ Facultad de Construcciones, Universidad de Oriente, Avenida de Céspedes 709 altos. Esq. N. Reparto Sueño, Santiago de Cuba, Cuba, ysocarrascordovi@gmail.com (Orcid:0000-0002-3198-3543)

${ }^{2}$ Facultad de Construcciones, Universidad de Oriente, Calle 6 Nº16. Reparto Santa Bárbara, Santiago de Cuba, Cuba, ealvarez@uo.edu.cu (Orcid:0000-0003-1819-263X)

El sistema prefabricado gran panel Soviético, ha mostrado un buen comportamiento sismorresistente en varios paises donde se ha implementado. Sin embargo, existen incertidumbres con las edificaciones construidas en la ciudad de Santiago de Cuba, la zona de mayor peligrosidad sísmica del país. Debido a que los códigos de diseño de la época de surgimiento del sistema prefabricado ya han sido derogados, unido a los daños patológicos y las transformaciones estructurales realizadas por los moradores. Por lo tanto, se requiere una verificación estructural, a través del chequeo de parámetros de control globales, las excentricidades de los centros de masas con respecto a los centros de rigidez, desplazamientos y derivas de los pisos, rigideces torsionales, los efectos P-A, entre otros aspectos. Para este análisis se escoge el edificio U-142-143, por tener condiciones críticas de explotación fundamentalmente. Se concluye que, aunque el sistema prefabricado, no cumple con todos los requerimientos actuales del diseño sismorresistente $y$ presentar significativas irregularidades en planta y elevación, el edificio analizado según las formulaciones en los códigos utilizados, puede conservar rigidez ante la acción sísmica. Una comparación entre el edificio según proyecto original y la variante actual, corrobora que los mayores cambios se observan en los periodos fundamentales. Consecuentemente hay variaciones en la deriva, desplazamientos y rigideces.

Palabras clave: verificación estructural, sistema prefabricado, irregularidades, rigidez, desplazamiento, derivas, excentricidades
The prefabricated Soviet great panel system has shown good earthquake resistance performance in several countries where it has been implemented. However, there are uncertainties with the buildings built in the city of Santiago de Cuba, the area of greatest seismic danger in the country. Due to the fact that the design codes of the time of the emergence of the prefabricated system have already been repealed, together with the pathological damages and structural transformations carried out by the inhabitants. Therefore, a structural verification is required, through the checking of global control parameters, the eccentricities of the centers of mass with respect to the centers of stiffness, displacements and drifts of the floors, torsional stiffness, the P-A effects, among other aspects. Building U-142-143 is chosen for this analysis, mainly because it has critical operating conditions. It is concluded that, although the prefabricated system does not comply with all the current requirements of earthquake resistant design and present significant irregularities in plan and elevation, the building analyzed according to the formulations in the codes used, can retain rigidity in the face of seismic action. A comparison between the building according to the original project and the current variant corroborates that the greatest changes are observed in the fundamental periods. Consequently there are variations in drift, displacement and stiffness.

Keywords: structural verification, precast system, irregularities, stiffness, displacement, drifts, eccentricities

\section{Introducción}

Desde 1964, el sistema prefabricado I-464, conocido popularmente en Cuba como gran panel Soviético, se convirtió en un recurso indispensable para resolver los problemas de viviendas. En un período de 26 años se 
erigieron solo en el municipio de Santiago de Cuba, 665 edificios, para un total de 769 en la provincia de igual nombre. Se construyeron edificios acordes a dos tipologías (con balcón y sin balcón) y de 4 o 5 niveles fundamentalmente. En la concepción de este sistema prefabricado, se manejaron criterios adecuados para ser implementado en zonas de alto peligro sísmico, aunque los códigos de diseño de la época de surgimiento del sistema prefabricado ya han sido derogados. El sistema de trasmisión de cargas es cruzado y las juntas tanto horizontales como verticales son húmedas, rígidas a nivel de superestructura. En edificaciones construidas en Chile y Armenia, con dicho sistema, se evidenció su buen comportamiento ante sismos de gran magnitud. Existen variados estudios de respuesta sísmica de estructuras prefabricadas de hormigón armado, basadas principalmente en ensayos de modelos en el laboratorio y enfocadas en las uniones y conexiones (e.g. Clough et al., 1989; Marcus y Thiers, 2015; Kurama et al., 2018). Sin embargo, son escasos los estudios sobre análisis sísmico de este tipo de estructuras afectadas por el deterioro, transformaciones y uso en el tiempo. Es por ello que este estudio aborda justamente esta temática de vital importancia.

Existen incertidumbres con las edificaciones construidas en Santiago de Cuba, a causa del deterioro patológico en los elementos estructurales y juntas entre ellos, así como las transformaciones estructurales que han realizados los moradores. Según Socarrás y Álvarez (2019), entre las transformaciones de peso, se encuentran los tanques de agua y paredes de mampostería. Como transformaciones de peso y rigidez, los rellenos de celosías de los paneles de fachada. Dentro de las transformaciones de rigidez, las aberturas o eliminación de paneles y losas.

Hasta el momento se han realizado investigaciones, que abarcan desde la caracterización de los materiales en las condiciones actuales de explotación hasta la predicción del comportamiento sísmico partiendo de la medición de los períodos de oscilación a través de las vibraciones ambientales $\left(T_{\mathrm{VA}}\right)$. Socarrás et al. $(2020 \mathrm{a}, \mathrm{b})$ obtienen en los elementos con deterioro patológico, una calidad pobre del hormigón y la resistencia a la compresión disminuye un $26 \%$ en relación a la prescrita en el proyecto original. Socarrás et al. (2020c) valoran la repercusión de algunas transformaciones estructurales. Socarrás et al. (2021a) concluyen que, en tres edificios instrumentados, los valores de $T_{\mathrm{VA}}$ se corresponden con períodos en el rango de los esperados ante la acción sísmica de diseño, por el deterioro de la rigidez. Socarrás et al. (2021b) por vía analítica, corroboran los resultados anteriores.

Uno de los edificios instrumentados, en estado crítico de explotación es el U-142-143 ubicado en el Distrito José Martí. Por eso, como punto de partida de una evaluación de la seguridad sísmica, urge realizar una verificación estructural en este edificio. Por lo tanto, se verifican parámetros de control globales, requerimientos actuales del diseño sismorresistente, excentricidades de los centros de masas con respecto a los centros de rigidez, desplazamientos y derivas de los pisos, rigideces torsionales, los efectos $\mathrm{P}-\Delta$, regularidad en planta y elevación.

\section{Materiales y métodos}

Se elaboran dos modelos multimasa del edificio U-142143 para el análisis dinámico con ETABS v18 (CSI, 2018), partiendo de las propiedades de los materiales, la geometría y vínculos de sus elementos componentes. Un modelo refleja el edificio según el proyecto original (Variante Original) y el otro modelo es del edificio en las condiciones actuales (Variante Actual).

Los paneles se consideran simplemente apoyados en la base y se modelan, al igual que las losas, como elementos finitos tipo shell, unidos de forma continua entre sí para producir un sistema estructural rígido y homogéneo conforme al detallado constructivo de las juntas. Igualmente se modelan las losas de escaleras como elementos finitos tipo shell conectadas a los paneles y las losas. Se asume que los elementos finitos shell tienen comportamiento membranal y de flexión como placas delgadas, empleando elementos finitos rectangulares con relación de forma adecuados. En la Figura 1 se aprecian los isométricos de los modelos geométricos, y en la Figura 2, las elevaciones de los tres ejes longitudinales y de los ejes transversales de la variante original.

El edificio U-142-143, es de la tipología 5 niveles sin balcón, con una longitud de $32 \mathrm{~m}$, ancho de $9.60 \mathrm{~m}$ y una altura de $14.33 \mathrm{~m}$. Los paneles interiores transversales son de $12 \mathrm{~cm}$ de espesor, espaciados a $3.20 \mathrm{~m}$ y los interiores longitudinales de $15 \mathrm{~cm}$ de espesor, espaciados a $4.80 \mathrm{~m}$. Todos los paneles exteriores en ambos sentidos, en su zona central, tienen $15 \mathrm{~cm}$ de espesor, pero en los bordes aumentan a $25 \mathrm{~cm}$. Las losas de entrepiso y cubierta son de espesor $12 \mathrm{~cm}$. En la variante actual, se redefine la geometría de las secciones de hormigón con daños patológicos, 


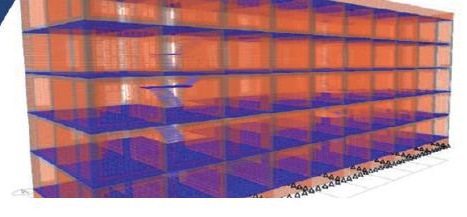

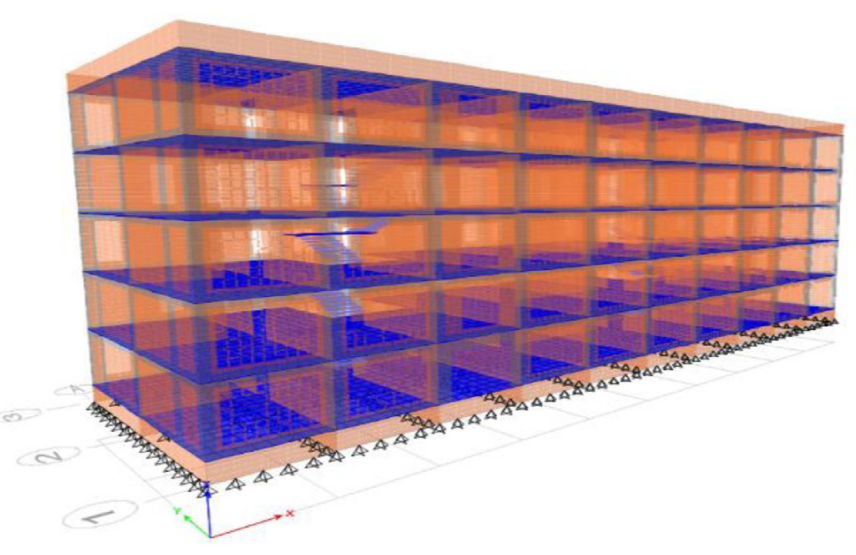

a)

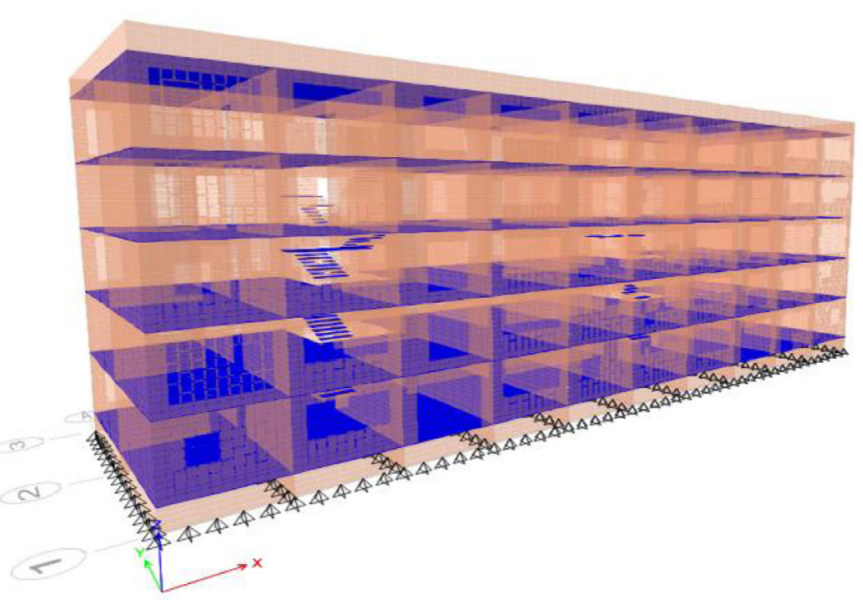

Figura 1: Modelos geométricos: a) variante original y b) variante actual

disminuyendo sus espesores un $25 \%$. La cimentación es corrida, con vigas fundidas in situ de sección rectangular sobre las cuales se colocan pequeños paneles llamados zócalos. Las resistencias de los materiales se obtienen de ensayos destructivos y no destructivos al hormigón y ensayos destructivos al acero (Socarrás, 2020; Socarrás et al., 2020a,b). Los resultados se resumen en la Tabla 1.

El módulo de elasticidad del hormigón prefabricado se calcula por la expresión recomendada por el ACI 318 (2019), pero con una reducción mayor del 40\%, según recomienda Lewicki (1968) para las edificaciones conformadas por paneles prefabricados por la presencia de las juntas. Por otra parte, se incrementa en un $20 \%$ por ser la acción sísmica de corta duración, totalizándose una penalización del 28\%. El módulo de cortante $G$ se obtiene del módulo del elasticidad $E$, asumiendo para el hormigón un coeficiente de Poisson $=0.17$. Para reflejar el

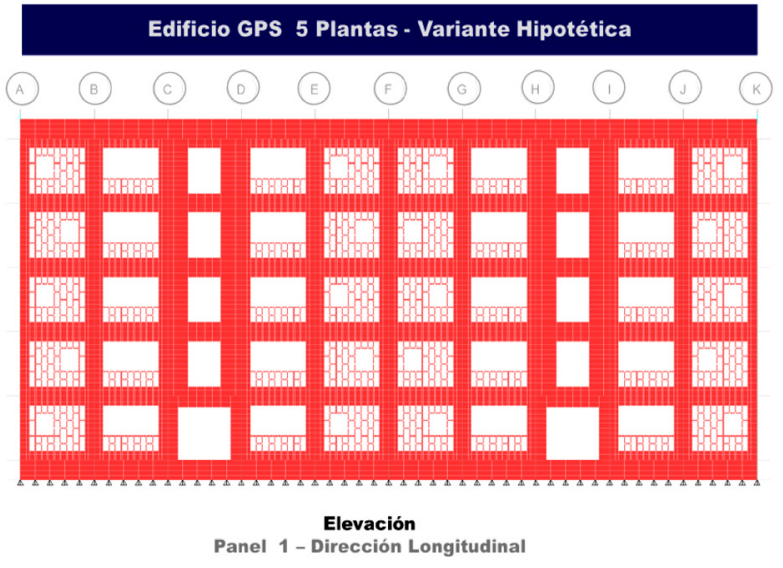

Edificio GPS 5 Plantas - Variante Hipotética

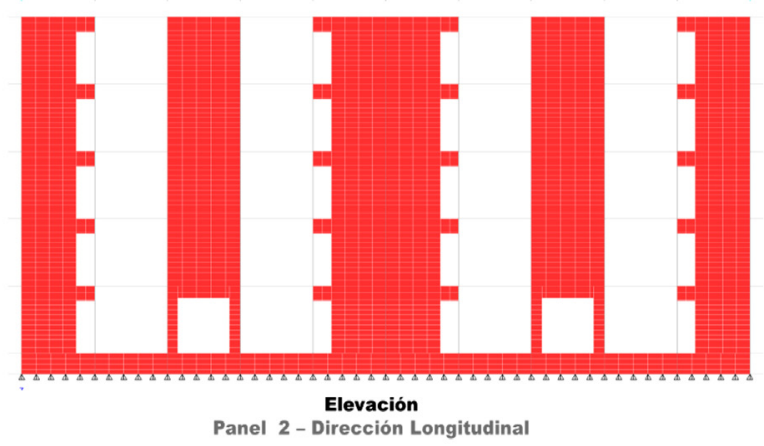

Edificio GPS 5 Plantas - Variante Hipotética
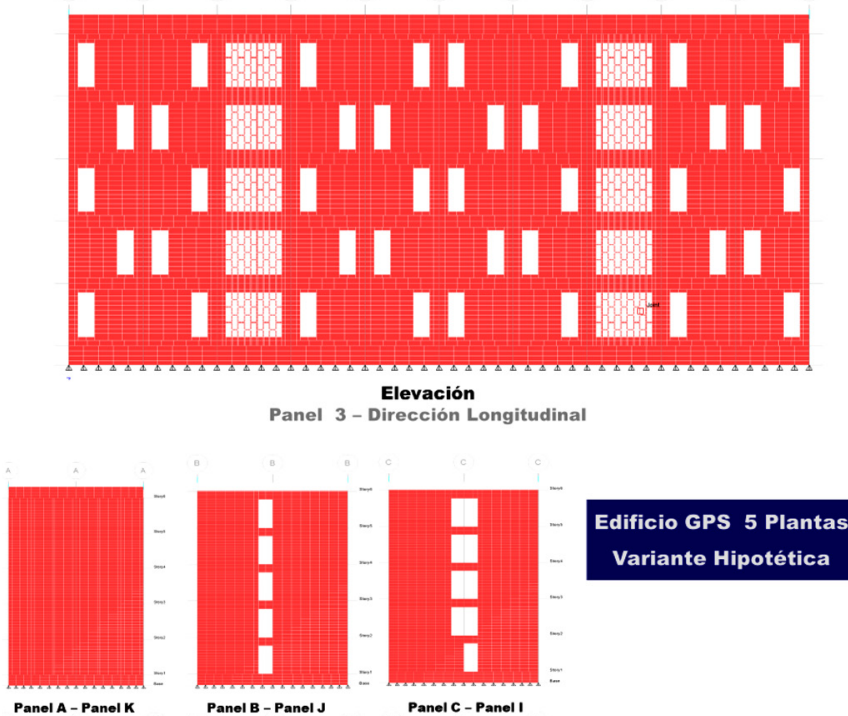

Variante Hipotética
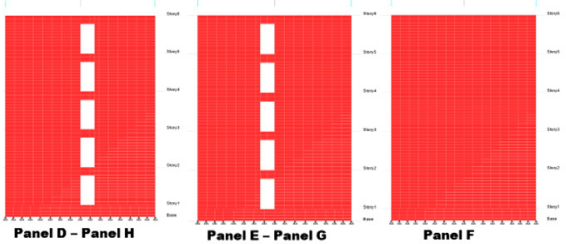

Elevación

Dirección Transversal

Figura 2: Elevaciones de ejes longitudinales y transversales. Variante Original 
Tabla 1: Características de los materiales

\begin{tabular}{|c|c|c|c|}
\hline Acero & $\begin{array}{l}\text { Diámetro, } \\
\text { mm }\end{array}$ & $\begin{array}{l}\text { Esfuerzo de } \\
\text { fluencia en } \\
\text { elementos } \\
\text { sin daños } \\
\text { patológicos } f \text {, } \\
\text { MPa }\end{array}$ & $\begin{array}{c}\text { Esfuerzo de } \\
\text { fluencia en } \\
\text { elementos } \\
\text { con daños } \\
\text { patológicos } f_{\mathrm{y}} \text {, } \\
\mathrm{MPa}\end{array}$ \\
\hline \multirow{2}{*}{ Corrugado } & 9.5 & 328.72 & 205.45 \\
\hline & 12 & 324.43 & 202.76 \\
\hline \multirow{3}{*}{ Liso } & 3 & 948.58 & 592.86 \\
\hline & 6 & 397.40 & 248.37 \\
\hline & 8 & 554.62 & 346.63 \\
\hline Hormigón & $\begin{array}{l}\text { Resistencia } \\
\text { a } \\
\text { compresión } \\
f_{\mathrm{c}}, \mathrm{MPa}\end{array}$ & \multicolumn{2}{|c|}{$\begin{array}{l}\text { Módulo de deformación } \\
\text { longitudinal } E, \mathrm{MPa}\end{array}$} \\
\hline $\begin{array}{l}\text { Prefabricado } \\
\text { Prefabricado } \\
\text { Relleno }\end{array}$ & $\begin{array}{l}16.00^{\mathrm{a}} \\
12.79^{\mathrm{b}} \\
10.00^{\mathrm{c}}\end{array}$ & $\begin{array}{l}135 \\
121 \\
107\end{array}$ & $\begin{array}{l}6.00^{\mathrm{a}} \\
2.23^{\mathrm{b}} \\
1.14^{\mathrm{c}}\end{array}$ \\
\hline
\end{tabular}

aHormigón prefabricado en elementos sin daños patológicos, bHormigón prefabricado en elementos con daños patológicos, 'Hormigón relleno de celosía

grado de fisuración y de acción inelástica que ocurre en los elementos inmediatamente antes de la fluencia, se emplean modificadores de la rigidez según FEMA 273 (1997) y ACI 318 (2019). En la Tabla 2 se resumen los modificadores de rigidez a flexión empleados. En la variante actual se obtuvieron de forma iterativa, considerando que $T_{\text {modelo }} \approx$ (1.02-1.15) $T_{\mathrm{VA}}$, como recomienda Socarrás (2020). Ver los valores de los períodos según las vibraciones ambientales $T_{\mathrm{VA}}$ en Socarrás et al. (2021a).

Tabla 2: Modificadores de rigidez a flexión en los modelos estructurales calibrados

\begin{tabular}{|l|c|c|}
\hline \multirow{2}{*}{ Elemento } & \multicolumn{2}{|c|}{ Modificadores de rigidez } \\
\cline { 2 - 3 } & $\begin{array}{c}\text { Variante } \\
\text { Original }\end{array}$ & $\begin{array}{c}\text { Variante } \\
\text { Actual }\end{array}$ \\
\hline $\begin{array}{l}\text { Paneles longitudinales interiores } \\
\text { y exteriores }\end{array}$ & $0.70 \mathrm{EI}$ & $0.35 \mathrm{EI}$ \\
\hline Paneles transversales exteriores & $0.70 \mathrm{EI}$ & $0.35 \mathrm{EI}$ \\
\hline Paneles transversales interiores & $0.70 \mathrm{EI}$ & $0.35 \mathrm{EI}$ \\
\hline $\begin{array}{l}\text { Paneles longitudinales y } \\
\text { transversales exteriores } \\
\text { interiores con daño patológico } \\
\text { severo }\end{array}$ & - & $0.15 \mathrm{EI}$ \\
\hline Losas celosías & $0.25 \mathrm{EI}$ & $0.25 \mathrm{EI}$ \\
\hline Losas con daño patológico severo & - & $0.10 \mathrm{EI}$ \\
\hline Relleno de celo & - & $0.15 \mathrm{EI}$ \\
\hline
\end{tabular}

Las cargas permanentes $(\mathrm{G})$ y de utilización $(\mathrm{Q})$ fueron definidas según las normas NC 283 (2003) y NC 284 (2003), respetando las consideraciones de los proyectos originales. Como carga permanente en la cubierta, tres capas de alfalto gravilla $\left(0.28 \mathrm{kN} / \mathrm{m}^{2}\right)$; en los entrepisos, relleno $(0.18 \mathrm{kN} /$ $\left.\mathrm{m}^{2} / \mathrm{cm}\right)$, mortero $\left(20.00 \mathrm{kN} / \mathrm{m}^{3}\right)$ y mosaico $\left(0.23 \mathrm{kN} / \mathrm{m}^{2} /\right.$ $\mathrm{cm})$. Como carga de uso en la cubierta, para desagüe por tragante $\left(2.00 \mathrm{kN} / \mathrm{m}^{2}\right)$ y en los entrepisos para habitaciones de viviendas comunes $\left(1.50 \mathrm{kN} / \mathrm{m}^{2}\right)$. El peso propio de los elementos es generado considerando el peso específico del material $\left(25 \mathrm{kN} / \mathrm{m}^{3}\right)$, que por ser prefabricado es mucho más compacto.

Se adicionan como cargas permanentes en la variante actual: tanques de agua en los patios de servicio, muro de mampostería en las áreas multipropósitos y relleno con hormigón de las celosías que tiene algunos paneles. Se modelan las cargas sísmicas (S) según la norma NC 46 (2017), con el Método del Espectro de Respuesta (MER) y el Método Estático Equivalente (MEE) utilizando los períodos fundamentales del análisis modal. Se consideran las tres componentes fundamentales de un sismo, los dos horizontales y la vertical, combinando el $100 \%$ de la carga sísmica en una de las direcciones principales, simultáneamente con el 30\% en las restantes direcciones. La carga sísmica en la dirección vertical se modela como un incremento de la carga permanente total que incluye el peso propio de la estructura. Este incremento se estima como el $20 \%$ de la carga permanente mencionada anteriormente por la aceleración de respuesta para un período corto determinado en el espectro de diseño para el perfil de suelo considerado.

También en cada uno de los pisos se consideraron las excentricidades accidentales de los centros de masas respecto a los centros de rigideces. Para el MER se utilizó como fórmula de superposición modal la CQC (complete quadratic combination), porque considera la proximidad de los modos en la respuesta, a través de los coeficientes de correlación modal. Se verifica que la suma de los factores de contribución modal sea la unidad, como propone Chopra (2014). Así debe verificarse que para todas las variantes se alcance una participación de al menos un $90 \%$, en este trabajo se aproximan al $80 \%$ de participación con 500 modos por variante. A partir de 500 modos se demuestra que sus contribuciones resultan despreciables por lo que 
se fija este valor con vistas a no incrementar los costos computacionales. El espectro de respuesta de diseño utilizado se elaboró para edificios de viviendas construidos en la ciudad de Santiago de Cuba, considerando la ubicación de los edificios estudiados y reducciones de las ordenadas espectrales para la disipación de energía del gran panel Soviético asumida en la investigación. A continuación, se detallan las consideraciones para la elaboración de dicho espectro.

Zona de peligro sísmico muy alto (5), donde las aceleraciones horizontales máximas del suelo $(0.30 \mathrm{~g})$ para el sismo de diseño se corresponden no solo con la zona sísmica sino también con la categoría de la obra. En el caso de edificios de viviendas, clasificados como "ordinarios", se recomienda un "sismo básico", el cual para períodos de vida útil de 50 años y una probabilidad de excedencia aceptada de un $10 \%$ se corresponden con un período de retorno de 475 años del sismo de diseño.

Tipo de suelo: perfil $\mathrm{D}$, asociados a suelos rígidos de cualquier espesor que cumpla con el criterio de velocidad de la onda de cortante $\left(180 \mathrm{~m} / \mathrm{s} \leq V_{\mathrm{s}} \leq 360 \mathrm{~m} / \mathrm{s}\right)$, o perfiles de suelos rígidos de cualquier espesor que cumpla cualquiera de las dos condiciones mostradas a continuación:

1) $15 \leq N \leq N$ número medio de golpes del ensayo 50 de penetración estándar, golpes/pie

2) $50 \mathrm{kPa} \leq s_{u}$ resistencia media al corte del ensayo $s_{u} \leq 100$ no drenado en los estratos de suelos $\stackrel{u}{\mathrm{kPa}}$ cohesivos

\section{Sistema estructural: E2 (sistema de muros)}

Factor de reducción por ductilidad $\mathrm{R}=1.5$, asumiendo respuesta cuasi elástica. Aunque la norma NC 46 (2017) recomienda hasta un valor de 4 , se valora que son estructuras prefabricadas proyectadas por códigos derogados, con poca ductilidad del acero de los elementos estructurales y un detallado inadecuado del refuerzo de las secciones de los elementos.

Las combinaciones de cargas empleadas son:

Combo 1: $\quad 1.2 \mathrm{G}+0.25 \mathrm{Q}+1.0 \mathrm{Sx}+0.3 \mathrm{Sy}+0.3 \mathrm{Sz}$

Combo 2: $\quad 1.2 \mathrm{G}+0.25 \mathrm{Q}+0.3 \mathrm{Sx}+1.0 \mathrm{Sy}+0.3 \mathrm{Sz}$

Combo 3: $\quad 1.2 \mathrm{G}+0.25 \mathrm{Q}+0.3 \mathrm{Sx}+0.3 \mathrm{Sy}+1.0 \mathrm{Sz}$

Combo 4: $\quad 0.9 \mathrm{G}+1.0 \mathrm{Sx}+0.3 \mathrm{Sy}+0.3 \mathrm{Sz}$

Combo 5: $\quad 0.9 \mathrm{G}+0.3 \mathrm{Sx}+1.0 \mathrm{Sy}+0.3 \mathrm{Sz}$

Combo 6: $\quad 0.9 \mathrm{G}+0.3 \mathrm{Sx}+0.3 \mathrm{Sy}+1.0 \mathrm{Sz}$

Combo 7: $\quad 1.2 \mathrm{G}+1.6 \mathrm{Q}$

\section{Resultados}

A continuación, se detalla el análisis de la verificación estructural en el sistema prefabricado gran panel Soviético. Se establecen las comparaciones entre la variante original y la variante actual. En la Tabla 3 se verifican los requerimientos actuales del diseño sismorresistente.

Tabla 3: Chequeo de requerimientos actuales del diseño sismorresistente en el sistema prefabricado gran panel Soviético

\begin{tabular}{|c|c|c|c|c|c|c|c|c|c|c|}
\hline $\begin{array}{c}\text { Espesor } \\
\text { elemento, } \\
\mathrm{mm}\end{array}$ & $\begin{array}{c}\text { Espesor } \\
\text { mínimo, } \\
\text { mm }\end{array}$ & $f_{\mathrm{c}}, \mathrm{MPa}$ & $\begin{array}{l}f_{\mathrm{c} \min }^{,}, \\
\mathrm{MPa}\end{array}$ & $\begin{array}{c}\text { Diámetros de } \\
\text { aceros, } \\
\text { mm }\end{array}$ & $f_{\mathrm{y}}, \mathrm{MPa}$ & \multicolumn{2}{|c|}{$\begin{array}{c}f_{\mathrm{y}} \\
\text { recomendada, } \mathrm{MPa}\end{array}$} & \multicolumn{3}{|c|}{$\begin{array}{c}\text { Espaciamiento } \\
\text { entre barras, } \\
\mathrm{mm}\end{array}$} \\
\hline \multicolumn{11}{|c|}{ Losa } \\
\hline \multirow{3}{*}{120} & \multirow{3}{*}{$140-250$} & \multirow{3}{*}{16.00} & \multirow{3}{*}{17.00} & 6 & 397.40 & Flexión & $\begin{array}{c}f_{\text {ymáx }} \\
550\end{array}$ & \multirow{3}{*}{$\begin{array}{c}150 \\
y \\
200\end{array}$} & Máx. & 240 \\
\hline & & & & 8 & 554.62 & Cortante & $f_{\text {ymáx }}$ & & \multirow{2}{*}{ Mín. } & \multirow{2}{*}{90} \\
\hline & & & & 9.5 & 328.72 & fricción & 420 & & & \\
\hline \multicolumn{11}{|c|}{ Panel } \\
\hline \multirow{2}{*}{$\begin{array}{c}120 \text { ó } \\
150\end{array}$} & \multirow{2}{*}{108} & \multirow{2}{*}{16.00} & \multirow{2}{*}{17.00} & 3 & 948.58 & \multirow{2}{*}{$\begin{array}{c}\text { Flexión, } \\
\text { axial } \\
\text { cortante }\end{array}$} & \multirow{2}{*}{$\begin{array}{l}f_{\text {ymáx }} \\
550\end{array}$} & 150 & \multirow{2}{*}{ Máx. } & \multirow{2}{*}{360} \\
\hline & & & & 12 & 324.43 & & & 200 & & \\
\hline
\end{tabular}


Tabla 4: Magnitudes de respuesta estructural en el edificio U-142-143

\begin{tabular}{|c|c|c|c|c|c|}
\hline \multicolumn{6}{|c|}{ Variante Original } \\
\hline \multirow{2}{*}{\multicolumn{3}{|c|}{ Magnitudes de respuestas }} & \multicolumn{3}{|c|}{ Método de cálculo } \\
\hline & & & & & $\begin{array}{c}\text { MER } \\
\text { (85\%del }\end{array}$ \\
\hline & & & & & \\
\hline $\begin{array}{l}\text { Peso del } \\
\text { edificio, } \\
\mathrm{kN}\end{array}$ & 19005.90 & $\begin{array}{c}\text { Cortante basal } \\
\text { longitudinal, } \\
\mathrm{kN}\end{array}$ & 8742.70 & 6603.0 & 7431.30 \\
\hline \begin{tabular}{|l|} 
Período \\
longitudi- \\
nal, s \\
\end{tabular} & 0.209 & \begin{tabular}{|c} 
Cortante basal \\
transversal, \\
$\mathrm{kN}$ \\
\end{tabular} & 8742.70 & 6145.11 & 7431.30 \\
\hline \begin{tabular}{|l|} 
Período \\
trans ver- \\
sal, s
\end{tabular} & 0.153 & $\begin{array}{c}\text { Coeficiente } \\
\text { sísmico longi- } \\
\text { tudinal }\end{array}$ & 0.46 & - & - \\
\hline $\begin{array}{l}\text { Período } \\
\text { de torsión, } \\
\mathrm{s}\end{array}$ & 0.130 & $\begin{array}{c}\text { Coeficiente } \\
\text { sísmico trans- } \\
\text { versal }\end{array}$ & 0.46 & - & - \\
\hline \multicolumn{6}{|c|}{ Variante Actual } \\
\hline \multicolumn{3}{|c|}{ Magnitudes de respuestas } & MEE & MER & $\begin{array}{c}\text { MER } \\
(85 \% \\
\text { del MEE } \\
\text { según } \\
\text { NC } \\
\text { 46:2017) }\end{array}$ \\
\hline $\begin{array}{l}\text { Peso del } \\
\text { edificio, } \\
\mathrm{kN}\end{array}$ & 20198.15 & $\begin{array}{c}\text { Cortante basal } \\
\text { longitudinal, } \\
\mathrm{kN}\end{array}$ & 9291.15 & 7573.40 & 7897.60 \\
\hline $\begin{array}{l}\text { Período } \\
\text { longitudi- } \\
\text { nal, s }\end{array}$ & 0.270 & $\begin{array}{c}\text { Cortante basal } \\
\text { transversal, } \\
\mathrm{kN}\end{array}$ & 9291.15 & 6847.20 & 7897.60 \\
\hline \begin{tabular}{|l|} 
Período \\
trans ver- \\
sal, s
\end{tabular} & 0.221 & $\begin{array}{c}\text { Coeficiente } \\
\text { sísmico longi- } \\
\text { tudinal }\end{array}$ & 0.46 & - & - \\
\hline $\begin{array}{l}\text { Período } \\
\text { de torsión, } \\
\mathrm{s}\end{array}$ & 0.188 & $\begin{array}{c}\text { Coeficiente } \\
\text { sísmico trans- } \\
\text { versal }\end{array}$ & 0.46 & - & - \\
\hline
\end{tabular}

En la Tabla 4 se muestran las magnitudes de respuesta estructural como los períodos fundamentales de oscilación, los cortantes basales y los coeficientes sísmicos; obtenidos con el MEE y el MER.

En la Tabla 5 y Figura 3 se muestra la variación de los centros de masa $\mathrm{CM}$ y centro de rigidez $\mathrm{CR}$, de la variante actual en relación a la variante original. Igualmente, en la Tabla 5 aparecen las excentricidades entre el CM y CR.

En la Tabla 6 se detallan los desplazamientos horizontales absolutos y relativos promedios para las dos direcciones
Tabla 5: Coordenadas y variación de los centros de masa y rigidez CM y CR, y excentricidades en el edificio U-142-143

\begin{tabular}{|c|c|c|c|c|c|c|c|c|}
\hline \multicolumn{9}{|c|}{ Centro de rigidez } \\
\hline & \multicolumn{2}{|c|}{$\begin{array}{l}\text { Variante } \\
\text { original }\end{array}$} & \multicolumn{2}{|c|}{ Variante actual } & \multicolumn{2}{|c|}{ Variación CR } & \multicolumn{2}{|c|}{ Variación CR } \\
\hline Piso & $\begin{array}{c}\mathrm{XCR}, \\
\mathrm{m}\end{array}$ & $\begin{array}{c}\mathrm{YCR}, \\
\mathrm{m}\end{array}$ & $\begin{array}{c}\mathrm{XCR}, \\
\mathrm{m}\end{array}$ & \begin{tabular}{|c|} 
YCR, \\
$\mathrm{m}$
\end{tabular} & $\mathrm{X}, \mathrm{m}$ & $\mathrm{Y}, \mathrm{m}$ & $\mathrm{X}, \%$ & $\mathrm{Y}, \%$ \\
\hline 5 & 16.040 & 8.794 & 16.337 & 7.255 & 0.298 & $\mid-1.539$ & .86 & -17 \\
\hline 4 & 16.031 & 8.602 & 16.372 & 7.052 & 0.340 & -1.550 & .12 & -18.02 \\
\hline 3 & 16.024 & 8.202 & 16.428 & 6.668 & 0.404 & -1.534 & 2.52 & -18.71 \\
\hline 2 & 16.024 & 7.701 & 16.522 & 6.187 & 08 & .514 & .17 & -19.66 \\
\hline 1 & 16.002 & 6.901 & 16.865 & 5.524 & 0.863 & $\mid-1.377$ & 5.39 & -19.96 \\
\hline 0 & 16.000 & 4.802 & 16.001 & 4.802 & 0.001 & 0.000 & 0. & 0.00 \\
\hline \multicolumn{9}{|c|}{ Centro de masa } \\
\hline & \multicolumn{2}{|c|}{$\begin{array}{l}\text { Variante } \\
\text { original }\end{array}$} & \multicolumn{2}{|c|}{ Variante actual } & \multicolumn{2}{|c|}{ Variación CM } & \multicolumn{2}{|c|}{ Variación CM } \\
\hline Piso & $\begin{array}{c}\mathrm{XCM}, \\
\mathrm{m}\end{array}$ & $\begin{array}{c}\mathrm{YCM}, \\
\mathrm{m}\end{array}$ & $\begin{array}{c}\mathrm{XCM}, \\
\mathrm{m}\end{array}$ & $\begin{array}{c}\mathrm{YCM}, \\
\mathrm{m}\end{array}$ & $\mathrm{X} m$ & $\mathrm{Y}, \mathrm{m}$ & $\mathrm{X}, \%$ & $\mathrm{Y}, \%$ \\
\hline 5 & 16.003 & 4.815 & 16.000 & 4.800 & -0.003 & 15 & -0.02 & -0.31 \\
\hline 4 & 15.965 & 4.448 & 15.968 & 4.220 & 0.003 & -0.228 & 0.02 & -5.13 \\
\hline 3 & 15.965 & 4.448 & 15.970 & 4.043 & 0.005 & -0.405 & 0.03 & -9.11 \\
\hline 2 & 15.965 & 4.448 & 15.970 & 4.023 & 55 & 25 & $0 \Omega$ & 055 \\
\hline 1 & 15.966 & 4.450 & 16.392 & 4.139 & 0.426 & -0.311 & 2.67 & -6.99 \\
\hline 0 & 15.967 & 4.641 & 16.402 & 4.331 & 0.435 & -0.310 & 2.72 & -6.68 \\
\hline \multicolumn{9}{|c|}{ Excentricidades en planta } \\
\hline & \multicolumn{2}{|c|}{$\begin{array}{l}\text { Variante } \\
\text { original }\end{array}$} & \multicolumn{2}{|c|}{ Variante actual } & \multicolumn{4}{|c|}{ Variación Excentricidad } \\
\hline Piso & Ex, m & Ey, m & Ex, $m$ & Ey, m & & & & \\
\hline 5 & -0.037 & -3.979 & -0.337 & -2.455 & & 1 & & \\
\hline 4 & -0.066 & -4.154 & -0.404 & -2.832 & & & & \\
\hline 3 & -0.059 & -3.754 & -0.457 & -2.625 & & & & \\
\hline 2 & -0.049 & -3.253 & -0.552 & -2.164 & & 03 & & 89 \\
\hline 1 & -0.036 & -2.451 & -0.473 & -1.385 & -0 . & 37 & & 66 \\
\hline 0 & -0.033 & -0.161 & 0.401 & -0.471 & & 434 & & 310 \\
\hline
\end{tabular}

principales de actuación del sismo y giros relativos de los pisos, obtenidos por el MEE. En la Figura 4 se comparan los desplazamientos entre las direcciones $\mathrm{X}$ e $\mathrm{Y}$ de variante original y la actual.

En la Tabla 7 aparecen las rigideces de los pisos a torsión estimadas con el MEE para la acción sísmica en la dirección de X. En la Figura 5 se observa el cambio, para cada uno de los pisos, de las rigideces relativas traslacionales y torsionales de la variante actual en relación a la original. 

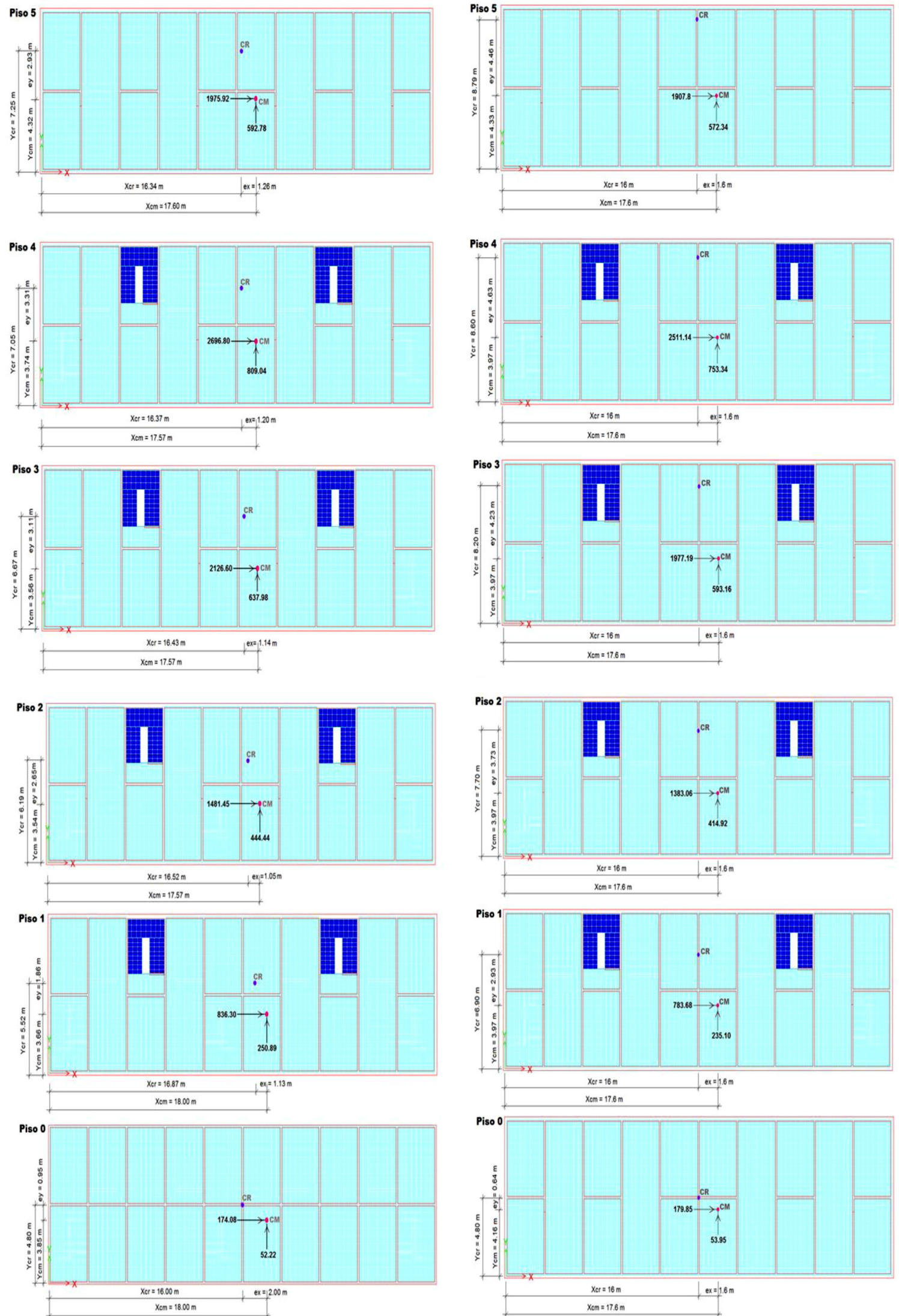

Figura 3: Posiciones de los centros de masa y rigidez. Variante actual y Variante original 
Tabla 6: Desplazamientos y derivas de los pisos en la variante original y actual del U-142-143.

\begin{tabular}{|c|c|c|c|c|c|}
\hline \multicolumn{6}{|c|}{ Variante Original } \\
\hline Piso & $\begin{array}{c}\text { Puntal } \\
\mathrm{m}\end{array}$ & $\begin{array}{c}\text { Deriva } \\
\mathrm{mm}\end{array}$ & $\begin{array}{c}\text { Desplaza- } \\
\text { miento } \\
\mathrm{mm}\end{array}$ & $\begin{array}{c}\text { Deriva } \\
\mathrm{mm}\end{array}$ & $\begin{array}{c}\text { Desplaza- } \\
\text { miento } \\
\mathrm{mm}\end{array}$ \\
\hline 5 & 13.50 & 0.824 & 7.157 & 0.791 & 4.629 \\
\hline 4 & 10.80 & 1.227 & 6.333 & 0.941 & 3.838 \\
\hline 3 & 8.10 & 1.543 & 5.106 & 1.022 & 2.897 \\
\hline 2 & 5.40 & 1.666 & 3.563 & 0.980 & 1.876 \\
\hline 1 & 2.70 & 1.624 & 1.898 & 0.768 & 0.896 \\
\hline \multicolumn{7}{|c|}{ Variante Actual } \\
\hline \multicolumn{7}{|c|}{ Dirección X } & Dirección Y \\
\hline Piso & $\begin{array}{c}\text { Puntal } \\
\mathrm{m}\end{array}$ & $\begin{array}{c}\text { Deriva } \\
\text { mm }\end{array}$ & $\begin{array}{c}\text { Desplaza- } \\
\text { miento } \\
\text { mm }\end{array}$ & $\begin{array}{c}\text { Deriva } \\
\text { mm }\end{array}$ & $\begin{array}{c}\text { Desplaza- } \\
\text { miento } \\
\text { mm }\end{array}$ \\
\hline 5 & 13.50 & 1.415 & 12.534 & 1.726 & 10.022 \\
\hline 4 & 10.80 & 2.210 & 11.119 & 2.057 & 8.297 \\
\hline 3 & 8.10 & 2.815 & 8.909 & 2.213 & 6.240 \\
\hline 2 & 5.40 & 2.961 & 6.094 & 2.115 & 4.028 \\
\hline 1 & 2.70 & 2.860 & 3.134 & 1.586 & 1.913 \\
\hline
\end{tabular}

Tabla 7: Rigidez torsional de cada uno de los pisos

\begin{tabular}{|c|c|c|c|}
\hline \multicolumn{4}{|c|}{ Variante Original } \\
\hline Piso & $\begin{array}{c}\text { Desplazamiento } \\
\text { angular, rad }\end{array}$ & $\begin{array}{c}\text { Drifts angular } \\
\text { rad }\end{array}$ & $\begin{array}{c}\text { Rigidez torsional } \\
\text { GNm/rad }\end{array}$ \\
\hline 5 & 0.000138 & 0.000026 & 327.19 \\
\hline 4 & 0.000112 & 0.000030 & 659.24 \\
\hline 3 & 0.000082 & 0.000031 & 851.00 \\
\hline 2 & 0.000051 & 0.000029 & 977.23 \\
\hline 1 & 0.000022 & 0.000021 & 1162.53 \\
\hline 0 & 0.000001 & 0.000001 & 6554.99 \\
\hline \multicolumn{4}{|c|}{ Variante Actual } \\
\hline 5 & 0.000179 & 0.000032 & 181.25 \\
\hline 4 & 0.000147 & 0.000040 & 358.23 \\
\hline 3 & 0.000107 & 0.000043 & 445.02 \\
\hline 2 & 0.000064 & 0.000038 & 520.73 \\
\hline 1 & 0.000026 & 0.000024 & 660.58 \\
\hline 0 & 0.000002 & 0.000002 & 4718.46 \\
\hline
\end{tabular}

La Tabla 8 muestra la verificación de la regularidad en planta y elevación según la norma NC 46 (2017). a)

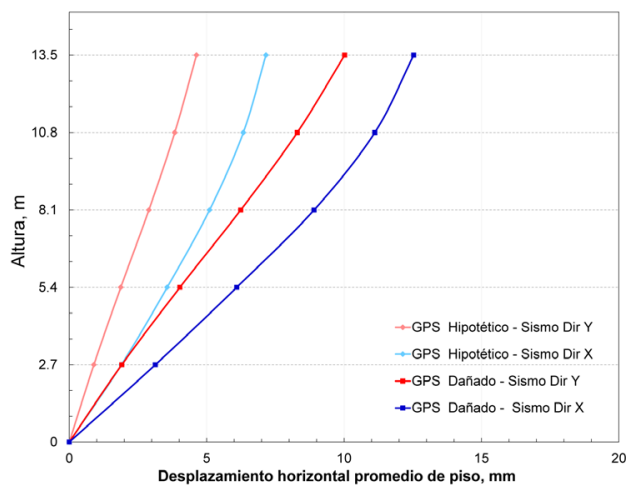

b)

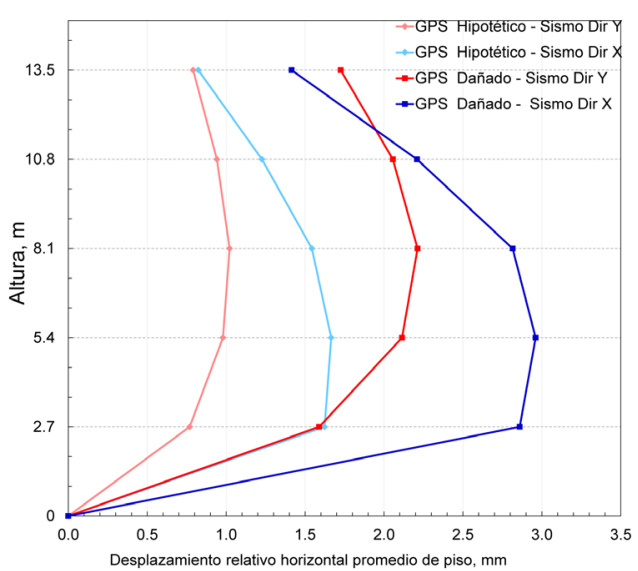

Figura 4: Comparación de desplazamientos entre direcciones X e Y. Variante Original Actual. a) Desplazamientos horizontales de piso y b) desplazamientos horizontales relativos

a)

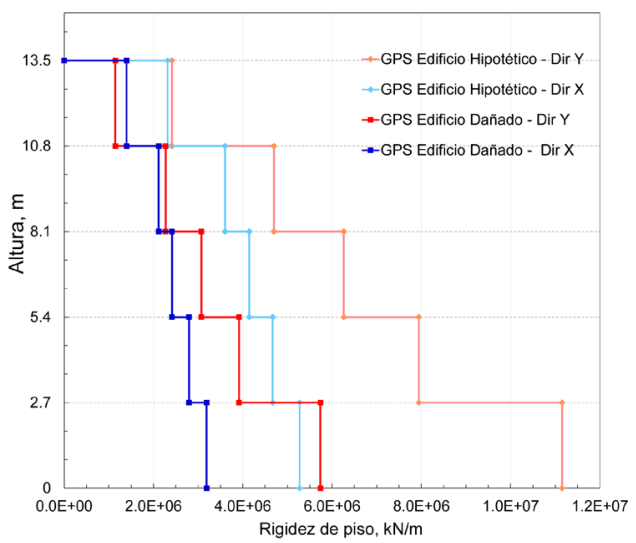

b)

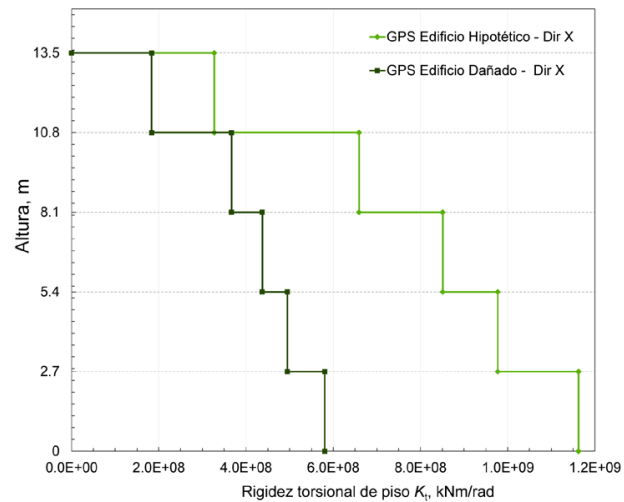

Figura 5: Cambio de la: a) rigidez de piso y b) rigidez torsional 
Tabla 8: Verificación de las irregularidades

\begin{tabular}{|c|c|c|c|c|c|c|c|c|c|c|}
\hline \multicolumn{11}{|c|}{ Irregularidad en elevación V1-A } \\
\hline \multicolumn{11}{|c|}{ Variante Original } \\
\hline & & & \multicolumn{4}{|c|}{$K_{\mathrm{i}} / K_{\mathrm{i}+1}$} & \multicolumn{4}{|c|}{$K_{\mathrm{i}} /\left(\left(K_{\mathrm{i}+1}+K_{\mathrm{i}+2}+K_{\mathrm{i}+3}\right) / 3\right)$} \\
\hline Piso & $\begin{array}{c}K_{x}, \\
\mathrm{MN} / \mathrm{m}\end{array}$ & $\begin{array}{c}K_{y}, \\
\mathrm{MN} / \mathrm{m}\end{array}$ & $\underset{\%}{\operatorname{Dir} X}$ & $\begin{array}{c}\text { Chequeo } \\
\geq 70 \%\end{array}$ & $\begin{array}{l}\operatorname{Dir} Y \\
\%\end{array}$ & $\begin{array}{c}\text { Chequeo } \\
\geq 70 \%\end{array}$ & $\begin{array}{c}\operatorname{Dir} X \\
\%\end{array}$ & $\begin{array}{c}\text { Chequeo } \\
\geq 80 \%\end{array}$ & $\begin{array}{c}\operatorname{Dir} Y \\
\%\end{array}$ & $\begin{array}{c}\text { Chequeo } \\
\geq 80 \%\end{array}$ \\
\hline 5 & 231.67 & 2411.88 & - & - & - & - & - & - & - & - \\
\hline 4 & 360.14 & 4696.01 & 155.5 & Cumple & 194.7 & Cumple & - & - & - & - \\
\hline 3 & 4145.26 & 6261.51 & 115.1 & Cumple & 133.3 & Cumple & - & - & - & - \\
\hline 2 & 4670.78 & 7942.00 & 112.7 & Cumple & 126.8 & Cumple & 139.2 & Cumple & 178.2 & Cumple \\
\hline 1 & 5274.33 & 11149.57 & 112.9 & Cumple & 140.4 & Cumple & 127.4 & Cumple & 177.0 & Cumple \\
\hline \multicolumn{11}{|c|}{ Variante Actual } \\
\hline 5 & 1396.41 & 1145.13 & - & - & - & - & - & - & - & - \\
\hline 4 & 2114.83 & 2272.17 & 151.5 & Cumple & 198.4 & Cumple & - & - & - & Cumple \\
\hline 3 & 2415.39 & 3073.14 & 114.2 & Cumple & 135.3 & Cumple & - & - & - & Cumple \\
\hline 2 & 2797.08 & 3916.18 & 115.8 & Cumple & 127.4 & Cumple & 141.6 & Cumple & 181.0 & Cumple \\
\hline 1 & 3188.35 & 5737.62 & 114.0 & Cumple & 146.5 & Cumple & 130.5 & Cumple & 185.9 & Cumple \\
\hline \multicolumn{11}{|c|}{ irregularidad en elevación V2 } \\
\hline \multicolumn{11}{|c|}{ Variante Original } \\
\hline \multirow{2}{*}{ Piso } & \multirow{2}{*}{$\begin{array}{l}W_{s}, \\
\mathrm{kN}\end{array}$} & \multicolumn{2}{|c|}{$W_{s i} / W_{s i-1} \leq 150 \%$} & \multicolumn{2}{|c|}{$W_{s i} / W_{s i+1} \leq 150 \%$} & & & & & \\
\hline & & $\%$ & Chequeo & $\%$ & Chequeo & & & & & \\
\hline 5 & 1300.42 & 69.51 & Cumple & - & - & & & & & \\
\hline 4 & 1870.72 & 99.94 & Cumple & 143.86 & Cumple & & & & & \\
\hline 3 & 1871.92 & 100.00 & Cumple & 100.06 & Cumple & & & & & \\
\hline 2 & 1871.92 & 99.89 & Cumple & 100.00 & Cumple & & & & & \\
\hline 1 & 1873.94 & 95.67 & Cumple & 100.11 & Cumple & & & & & \\
\hline 0 & 1958.73 & - & - & 104.52 & Cumple & & & & & \\
\hline \multicolumn{11}{|c|}{ Variante Actual } \\
\hline 5 & 1300.42 & 64.29 & Cumple & - & - & & & & & \\
\hline 4 & 2022.58 & 93.05 & Cumple & 155.53 & $\begin{array}{c}\text { No } \\
\text { cumple }\end{array}$ & & & & & \\
\hline 3 & 2173.59 & 101.21 & Cumple & 107.47 & Cumple & & & & & \\
\hline 2 & 2147.66 & 101.63 & Cumple & 98.81 & Cumple & & & & & \\
\hline 1 & 2113.15 & 96.73 & Cumple & 98.39 & Cumple & & & & & \\
\hline 0 & 2184.51 & - & - & 103.38 & Cumple & & & & & \\
\hline \multicolumn{11}{|c|}{ irregularidad en planta $\mathrm{H} 1-\mathrm{A}$} \\
\hline \multicolumn{11}{|c|}{ Variante Original } \\
\hline & \multicolumn{3}{|c|}{$($ Drift $\max /$ Drift $\min ) \leq 1.5$} & \multicolumn{3}{|c|}{$($ Drift $\max /$ Drift $\min ) \leq 1.5$} & & & & \\
\hline & Variación & Variación & & Variación & Variación & & & & & \\
\hline Piso & $\begin{array}{c}\text { de deriva } \\
\text { Dir. X } \\
\text { mm }\end{array}$ & $\begin{array}{c}\text { vs } \\
\text { valor min } \\
\text { \#veces }\end{array}$ & Chequeo & $\begin{array}{c}\text { de deriva } \\
\text { Dir. Y } \\
\text { mm }\end{array}$ & $\begin{array}{c}\text { vs } \\
\text { valor min } \\
\text { \#veces }\end{array}$ & Chequeo & & & & \\
\hline 5 & -0.221 & 1.31 & Cumple & 0.268 & 1.41 & Cumple & & & & \\
\hline 4 & -0.248 & 1.22 & Cumple & 0.328 & 1.42 & Cumple & & & & \\
\hline 3 & -0.254 & 1.18 & Cumple & 0.365 & 1.44 & Cumple & & & & \\
\hline 2 & -0.233 & 1.15 & Cumple & 0.359 & 1.45 & Cumple & & & & \\
\hline 1 & -0.169 & 1.11 & Cumple & 0.308 & 1.50 & Cumple & & & & \\
\hline \multicolumn{11}{|c|}{ Variante Actual } \\
\hline 5 & -0.234 & 1.18 & Cumple & -0.431 & 1.29 & Cumple & & & & \\
\hline 4 & -0.273 & 1.13 & Cumple & -0.559 & 1.31 & Cumple & & & & \\
\hline 3 & -0.290 & 1.11 & Cumple & -0.647 & 1.34 & Cumple & & & & \\
\hline 2 & -0.235 & 1.08 & Cumple & -0.605 & 1.33 & Cumple & & & & \\
\hline 1 & -0.113 & 1.04 & Cumple & -0.340 & 1.24 & Cumple & & & & \\
\hline
\end{tabular}

$K_{x}, K_{v}$ : rigidez en relación a $x$ o $y ; K_{i}$ : rigidez del nivel $i ; W_{s}$ : peso sísmico; $W_{s i}$ : peso sísmico del nivel $i$ 
En la Tabla 9 se verifica con el MEE el efecto P- $\Delta$ para las dos direcciones principales de actuación del sismo según la norma NC 46 (2017). Los valores de los coeficientes $C_{\mathrm{d}}$ y $\beta$ son 1.5 y 1.0 respectivamente, para todos los casos.

\section{Análisis de resultados}

Al verificar las propiedades de los elementos prefabricados que conforman la edificación con los requerimientos actuales del diseño sismorresistente según el ACI 318 (2019), resumidos en la Tabla 3, se concluye que no se cumple con los espesores mínimos requeridos para las losas y paneles, así como el detallado del acero en los paneles es inadecuado, debido a que no tienen cercos de confinamiento de la sección transversal para unir las dos mallas. FEMA 273 (1997) y FEMA 310 (1998) exponen que la aparición de los daños sísmicos potenciales en las estructuras prefabricadas de paneles, es por la capacidad insuficiente a cortante y/o flexión de los paneles, falta de confinamiento en sus bordes, longitud de empalme inadecuada para el refuerzo longitudinal, aberturas muy

Tabla 9: Verificación de los efectos P- $\Delta$

\begin{tabular}{|c|c|c|c|c|c|c|c|c|}
\hline \multicolumn{9}{|c|}{ Variante Original } \\
\hline Piso & $\begin{array}{c}\text { Dir - X } \\
\text { P, kN }\end{array}$ & $\begin{array}{l}\text { Dir - X } \\
\mathrm{V}, \mathrm{kN}\end{array}$ & $\begin{array}{c}\text { Dir - X } \\
\text { Drifts, m }\end{array}$ & $\begin{array}{c}\text { Puntal } \\
\mathrm{m}\end{array}$ & $\begin{array}{c}\text { Dir - X } \\
\text { CitaAdim }\end{array}$ & $\begin{array}{c}\text { Valor max } \\
0.5 /\left(\beta C_{\mathrm{d}}\right) \\
\text { Cita Adim. }\end{array}$ & $\begin{array}{c}\text { Dir - X } \\
\text { Cita máx } \\
\text { Adim. }\end{array}$ & $\begin{array}{c}\text { Estabilidad } \\
\text { Dir }-X\end{array}$ \\
\hline 5 & 1632.95 & 1907.80 & 0.000791 & 2.70 & 0.00025 & 0.33 & 0.25 & Cumple \\
\hline 4 & 5000.72 & 4418.94 & 0.000941 & 2.70 & 0.00039 & 0.33 & 0.25 & Cumple \\
\hline 3 & 8419.34 & 6396.13 & 0.001022 & 2.70 & 0.00050 & 0.33 & 0.25 & Cumple \\
\hline 2 & 11847.07 & 7779.19 & 0.000980 & 2.70 & 0.00055 & 0.33 & 0.25 & Cumple \\
\hline 1 & 15285.69 & 8562.87 & 0.000768 & 2.70 & 0.00051 & 0.33 & 0.25 & Cumple \\
\hline Piso & $\begin{array}{c}\text { Dir - Y } \\
P, k N\end{array}$ & $\begin{array}{c}\text { Dir - Y } \\
\mathrm{V}, \mathrm{kN}\end{array}$ & $\begin{array}{c}\text { Dir - Y } \\
\text { Drifts, m }\end{array}$ & $\begin{array}{c}\text { Puntal } \\
\mathrm{m}\end{array}$ & $\begin{array}{c}\text { Dir - Y } \\
\text { CitaAdim. }\end{array}$ & $\begin{array}{c}\text { Valor max } \\
0.5 /\left(\beta C_{\mathrm{d}}\right) \\
\text { Cita Adim. }\end{array}$ & $\begin{array}{c}\text { Dir - Y } \\
\text { Cita máx } \\
\text { Adim. }\end{array}$ & $\begin{array}{c}\text { Estabilidad } \\
\text { Dir - Y }\end{array}$ \\
\hline 5 & 1632.95 & 1907.80 & 0.000824 & 2.70 & 0.00026 & 0.33 & 0.25 & Cumple \\
\hline 4 & 5000.72 & 4418.94 & 0.001227 & 2.70 & 0.00051 & 0.33 & 0.25 & Cumple \\
\hline 3 & 8419.34 & 6396.13 & 0.001543 & 2.70 & 0.00075 & 0.33 & 0.25 & Cumple \\
\hline 2 & 11847.07 & 7779.19 & 0.001666 & 2.70 & 0.00094 & 0.33 & 0.25 & Cumple \\
\hline 1 & 15285.69 & 8562.87 & 0.001624 & 2.70 & 0.00107 & 0.33 & 0.25 & Cumple \\
\hline \multicolumn{9}{|c|}{ Variante Actual } \\
\hline Piso & $\begin{array}{c}\text { Dir - X } \\
P, k N\end{array}$ & $\begin{array}{l}\text { Dir - X } \\
\mathrm{V}, \mathrm{kN}\end{array}$ & $\begin{array}{c}\text { Dir - X } \\
\text { Drifts, m }\end{array}$ & $\begin{array}{l}\text { Puntal } \\
\mathrm{m}\end{array}$ & $\begin{array}{c}\text { Dir - X } \\
\text { CitaAdim. }\end{array}$ & $\begin{array}{c}\text { Valor max } \\
0.5 /\left(\beta C_{\mathrm{d}}\right) \\
\text { Cita Adim. }\end{array}$ & $\begin{array}{l}\text { Dir - X } \\
\text { Cita máx } \\
\text { Adim. }\end{array}$ & $\begin{array}{c}\text { Estabilidad } \\
\text { Dir - X }\end{array}$ \\
\hline 5 & 1632.95 & 1975.92 & 0.001415 & 2.70 & 0.00043 & 0.33 & 0.25 & Cumple \\
\hline 4 & 5162.33 & 4672.72 & 0.002210 & 2.70 & 0.00090 & 0.33 & 0.25 & Cumple \\
\hline 3 & 8905.85 & 6799.32 & 0.002815 & 2.70 & 0.00137 & 0.33 & 0.25 & Cumple \\
\hline 2 & 12626.83 & 8280.77 & 0.002961 & 2.70 & 0.00167 & 0.33 & 0.25 & Cumple \\
\hline 1 & 16318.54 & 9117.07 & 0.002860 & 2.70 & 0.00190 & 0.33 & 0.25 & Cumple \\
\hline Piso & $\begin{array}{c}\text { Dir - Y } \\
\mathrm{P}, \mathrm{kN}\end{array}$ & $\begin{array}{l}\text { Dir - Y } \\
\mathrm{V}, \mathrm{kN}\end{array}$ & $\begin{array}{c}\text { Dir - Y } \\
\text { Drifts, m }\end{array}$ & $\begin{array}{c}\text { Puntal } \\
\mathrm{m}\end{array}$ & $\begin{array}{c}\text { Dir - Y } \\
\text { CitaAdim. }\end{array}$ & $\begin{array}{c}\text { Valor max } \\
0.5 /\left(\beta C_{d}\right) \\
\text { Cita Adim. }\end{array}$ & $\begin{array}{c}\text { Dir - Y } \\
\text { Cita máx } \\
\text { Adim. }\end{array}$ & $\begin{array}{c}\text { Estabilidad } \\
\text { Dir - Y }\end{array}$ \\
\hline 5 & 1632.95 & 1975.92 & 0.001726 & 2.70 & 0.00053 & 0.33 & 0.25 & Cumple \\
\hline 4 & 5162.33 & 4672.72 & 0.002057 & 2.70 & 0.00084 & 0.33 & 0.25 & Cumple \\
\hline 3 & 8905.85 & 6799.32 & 0.002213 & 2.70 & 0.00107 & 0.33 & 0.25 & Cumple \\
\hline 2 & 12626.83 & 8280.77 & 0.002115 & 2.70 & 0.00119 & 0.33 & 0.25 & Cumple \\
\hline 1 & 16318.54 & 9117.07 & 0.001589 & 2.70 & 0.00105 & 0.33 & 0.25 & Cumple \\
\hline
\end{tabular}

Cita Adim: coeficiente de inestabilidad adimensional 
grandes, espesores insuficientes y conexiones inadecuadas. Por lo tanto, el sistema muestra vulnerabilidades intrínsecas a su concepción.

Tampoco se cumple con la calidad del hormigón, ni con la calidad del acero para las barras de diámetro $3 \mathrm{~mm}$, que conforman las mallas electrosoldadas de los paneles. Estas barras de $3 \mathrm{~mm}$ tienen, además, un esfuerzo de fluencia superior al recomendado, y son barras lisas con un comportamiento no dúctil, al no poseer un escalón de fluencia definido, como se observa en Socarrás (2020). Carrillo y Alcocer (2013) observaron en sus ensayos, la fractura súbita de mallas electrosoldadas, argumentando que esto conduce a un modo de falla frágil e indeseable. Por eso, plantean que para el diseño sísmico de muros cuyo refuerzo a cortante en el alma es con malla electrosoldada de aceros de poca ductilidad, la reducción de la cuantía de refuerzo en proporción al aumento del esfuerzo de fluencia no se debe permitir por los reglamentos.

Como en los paneles también se usan aceros dúctiles de 12 $\mathrm{mm}$, la poca reserva de ductilidad del sistema, dependerá en alguna medida del estado tenso-deformacional a que estén sometidos estos aceros debido a las acciones sísmicas de cálculo. No obstante, se mantiene la hipótesis de que un adecuado comportamiento sísmico estaría en correspondencia con un comportamiento cuasielástico, como justifica el factor de reducción de ductilidad natural asumido de 1.5.

Cuando se valoran los parámetros de control global de la Tabla 4, se aprecian cambios en relación a la variante original, con los mayores incrementos en los períodos fundamentales. Al comparar los períodos fundamentales de la variante actual, con el empírico determinado por Oliva (2001), se evalúan incrementos del $63.6 \%$ en el período longitudinal; $33.9 \%$ y $13.9 \%$ en los períodos transversal y de torsión, respectivamente. El aumento de los períodos se debe tanto a la disminución de rigideces por la presencia de daños patológicos, como al incremento del peso sísmico por las modificaciones realizadas por los moradores.

Igualmente son mayores los períodos longitudinales en ambas variantes, en relación a los períodos transversales. Esto evidencia que la edificación tiene menor rigidez en la dirección longitudinal, incluso en la variante original. Socarrás et al. (2021a) argumentan que: ... teniendo en cuenta, la relación de las dimensiones de largo (16 m) y ancho (9.6 m) de las edificaciones, estas deberían tener mayor rigidez en la dirección longitudinal y, por tanto, menores valores de los períodos fundamentales longitudinales. Sin embargo, tienen mayor rigidez en la dirección transversal por ser el área de paneles longitudinales en todos los niveles menor que el área de paneles transversales."

Por otro lado, los cortantes basales longitudinales y transversales sufren ligeros incrementos en la variante actual, aumentan por el incremento del peso sísmico, no por el incremento de los períodos longitudinales y transversales; porque estos son mayores que el período de esquina $\left(T_{1}\right)$ y se mantienen en la meseta del espectro. Por eso los coeficientes sísmicos por el MEE, se mantienen constantes en la dirección longitudinal y transversal. Los incrementos del cortante basal, están en el orden del $6 \%$ en la dirección longitudinal y del $15 \%$ en la transversal.

Se observa en la Figura 3 y Tabla 5, que el edificio en la variante original tiene excentricidades en planta, significativas en la dirección de $\mathrm{Y}$ a causa que los paneles de la fachada posterior (eje 3) tienen menos ventanas que los de la fachada principal (eje 1) e incluso que los paneles del eje intermedio (eje 2) debilitados por la presencia de grandes vanos para lograr espacios multifuncionales.

En la variante actual se perciben cambios en la posición de los CM, CR y en las excentricidades resultantes respecto a la variante original. Las variaciones en la posición de los $\mathrm{CR}$ son más significativas que aquellas de los CM, estas últimas a causa de las transformaciones de peso realizadas por los moradores, en el caso de los rellenos de celosías también causan modificaciones en los CR conjuntamente con los daños patológicos. El incremento de la rigidez de los paneles de fachada debido a los rellenos de celosías, conduce a que, en la variante actual, el CR se aleje del eje 3 (eje de mayor rigidez) reduciéndose las excentricidades en la dirección Y, por lo que en consecuencia se incrementen las solicitaciones sísmicas en dichos paneles de fachada no aparejado con un incremento de su resistencia. Pero como esta rigidez es "instantánea" por los materiales informales que se usan y la forma de colocación de las celosías unido a un daño patológico concentrado en estos paneles, se condiciona aún más su riesgo de fallo.

Luego se estiman los resultados de las Tablas 6 y 7 , así como 
de las Figuras 4 y 5 . Tanto en la variante original, como en la actual, las rigideces en la dirección $\mathrm{X}$ son menores (del primer al cuarto nivel), que en Y. Las rigideces sufren disminuciones en la variante actual respecto a la original de $48.5 \%$ en $\mathrm{X}, 39.5 \%$ en Y y la torsional de un $44.6 \%$. Consecuentemente, los desplazamientos en la dirección $\mathrm{X}$ son mayores, con un $65 \%$ superior que la variante original. Sin embargo, los desplazamientos se incrementan más en la dirección Y (113\%). A la par, los desplazamientos relativos son mayores en la dirección $\mathrm{Y}$, solo para el quinto nivel. A pesar de los incrementos de desplazamientos, la deriva es menor que la admisible ofrecida por la norma NC 46 (2017).

La valoración de la deriva puede ser más conservadora teniendo en cuenta que el refuerzo de los paneles es con mallas electrosoldadas. Carrillo y Alcocer (2013) proponen que, los factores de seguridad para los niveles de deriva permitidos deben ser más altos en los muros con mallas electrosoldadas que los utilizados para los muros reforzados con barras corrugadas de bajo carbono. A causa que, la capacidad de desplazamiento puede estar limitada por la baja capacidad de alargamiento del alambre de refuerzo estirado en frío. Esto implica que la deriva admisible debe ser menor, lo que conlleva evidentemente a un análisis más conservador.

En la evaluación de los desplazamientos, se deben atender los resultados de López y Music (2016). Dichos autores, obtienen desplazamientos considerando modificadores de rigidez a flexión, con valores inferiores, al desplazamiento calculado según la ordenada del espectro elástico de desplazamiento para un 5\% de amortiguamiento respecto del crítico, correspondiente al período agrietado de mayor masa traslacional en la dirección analizada multiplicada por 1.3, como regula el decreto DS61 (2011). En esta investigación que aborda la revisión estructural de las edificaciones existentes, al emplear modificadores de rigidez a flexión se obtienen desplazamientos menos conservadores que aquellos determinados por formulaciones aproximadas en reglamentos sísmicos. Esto conduce a mayor precisión en el chequeo de estados límites que dependen de la rigidez del sistema.

Teniendo en cuenta los resultados de la Tabla 8, se justifica el empleo del MEE como calibrador de los modelos estructurales. A pesar de que el edificio objeto de estudio posee significativas excentricidades en planta en la dirección del eje $\mathrm{Y}$, tiene grandes rigideces torsionales de los pisos en relación con sus rigideces laterales. Esto condiciona que los giros de los pisos con respecto a los CR alcancen valores pequeños que garantiza que se cumpla lo especificado en la NC46 (2017), en relación a las irregularidades H1A, V1A y V2. Finalmente, del análisis de la Tabla 9, se obtiene que los efectos P- $\Delta$, se pueden despreciar, ya que se cumple con la verificación de estabilidad. Aunque en el edificio en las condiciones actuales de explotación el margen de seguridad es mucho menor.

\section{Conclusiones}

El sistema prefabricado gran panel Soviético muestra vulnerabilidades intrínsecas a su concepción, ya que no cumple con todos los requerimientos actuales del diseño sismorresistente. Los espesores mínimos requeridos para las losas y paneles, así como el detallado del acero en los paneles, son inadecuado, no obedecen al diseño conceptual. Tampoco se cumple con la calidad del hormigón, ni con la calidad del acero para las barras de diámetro $3 \mathrm{~mm}$ que carecen de un escalón de fluencia definido. Lo anterior condiciona que un buen comportamiento sísmico solo sería posible para estados tensionales que garanticen un comportamiento cuasielástico de sus elementos estructurales. El edificio analizado en las condiciones actuales de explotación evidencia los mayores incrementos en los períodos fundamentales de oscilación traslacionales y de torsión. Esto se debe tanto a la disminución de rigideces por la presencia de daños patológicos, como al incremento del peso sísmico por la colocación de tanques de agua y adición de paredes de mampostería.

A pesar de que el edificio objeto de estudio posee significativas excentricidades en planta en la dirección del eje $\mathrm{Y}$, tiene grandes rigideces torsionales de los pisos en relación con sus rigideces laterales. Esto condiciona que los giros de los pisos con respecto a los CR alcancen valores pequeños que garantiza que se cumpla lo especificado en la NC 46 (2017), en relación a las irregularidades H1A, V1A y V2, justificándose el empleo del MEE como calibrador de los modelos estructurales. Se aprecia también, variaciones en la posición del centro de masa, centro de rigidez y en sus correspondientes excentricidades con relación a la variante original. Específicamente las rigideces 
sufren disminuciones significativas incrementándose los desplazamientos laterales y giros de los pisos. Aunque los desplazamientos laterales se mantienen en los rangos admisibles, cumpliéndose especificaciones relacionadas a la rigidez del sistema, el margen de seguridad es mucho menor. El edificio U-142-143, aún en las condiciones críticas de explotación puede conservar rigidez ante la acción sísmica, pero se impone, la necesidad de realizar una verificación de la capacidad resistente de los elementos estructurales y evaluación de su desempeño, sobre todo por la redistribución de esfuerzos por el cambio de la posición del centro de rigidez, así como los daños patológicos presentes.

La investigación realizada permite concluir que debe prestarse especial atención a la evolución de los daños patológicos por su incidencia en la verificación estructural de aquellas especificaciones que dependen de la rigidez del sistema. Aún en las condiciones críticas de explotación puede conservar rigidez ante la acción sísmica. Aunque se impone la necesidad de evaluar la seguridad sísmica, a través de la verificación de la capacidad resistente de los elementos y juntas estructurales, sobre todo por la redistribución de esfuerzos por el cambio de la posición del centro de rigidez y de masa, así como los daños patológicos presentes.

\section{Referencias}

ACI 318 (2019). Building code requirements for structural concrete. American Concrete Institute ACI. Washington DC, USA

Carrillo, J. and Alcocer, S.M. (2013). Shear strength of reinforced concrete walls for seismic design of low-rise housing. ACI Structural Journal 110(3), 415-426

Chopra, A. (2014). Dinámica de estructuras. Pearson Educación, México

Clough, R.W., Malhas, F. and Oliva, M.G. (1989). Seismic behavior of large panel precast concrete walls: analysis and experiment. PCI Journal 34(2), 42-66

CSI (2018). ETABS v18. Computers \& Structures, Inc. CSI. Structural and earthquake engineering software. USA
DS61 (2011). Decreto Supremo Nº61. Reglamento que fija el diseño sísmico de edificios. Ministerio de Vivienda y Urbanismo, Santiago, Chile

FEMA 310 (1998). Handbook for the seismic evaluation of buildings. Federal Emergency Management Agency FEMA. Washington DC, USA

FEMA P-154 (2015). Rapid visual screening of buildings for potential seismic hazards: A Handbook. Federal Emergency Management Agency FEMA. Washington DC, USA

FEMA 273 (1997). NEHRP guidelines for the seismic rehabilitation of buildings. Federal Emergency Management Agency FEMA, Washington DC, USA

Kurama, Y.C., Sritharan, S., Fleischman, R.B., Restrepo, J.I., Henry, R.S., Cleland, N.M., Ghosh, S.K. and Bonelli, P. (2018). Seismic-resistant precast concrete structures: state of the art. Journal of Structural Engineering 144(4), p.03118001.

Lewicki, B. (1968). Edificios de viviendas prefabricadas con elementos de grandes dimensiones. Arkady, Polonia

López, C. y Music, J. (2016). Análisis del período y desplazamiento de edificios de hormigón armado considerando distintos grados de rigidez en sus elementos resistentes. Obras y Proyectos 19, 33-47

Marcus, J. y Thiers, R. (2015). Control del daño sísmico estructural en pórticos prefabricados de hormigón armado a través de uniones híbridas autocentrantes. Obras y Proyectos $\mathbf{1 8}$, 46-55

NC 283 (2003). Densidad de materiales naturales, artificiales y de elementos de construcción como carga de diseño. Comité Estatal de Normalización, La Habana, Cuba

NC 284 (2003). Edificaciones. Cargas de uso. Comité Estatal de Normalización, La Habana, Cuba

NC 46 (2017). Construcciones sismo resistentes. Requisitos básicos para el diseño y construcción. Comité Estatal de Normalización, La Habana, Cuba

Oliva, R. (2001). Determinación experimental del periodo fundamental de vibración de estructuras para la evaluación de la vulnerabilidad en Cuba. Grupo de Ingeniería Sísmica. Centro Nacional de Investigaciones Sismológicas. Cuba 
Socarrás, Y.C. y Álvarez, E. (2019). Factores causantes de daños potenciales en el Gran Panel Soviético. VI Jornada Internacional de Ingeniería Civil. Holguín, Cuba

Socarrás-Cordoví, Y.C., González-Diaz, 1., Alvarez-Deulofeu, E., González -Fernández, M.M., Roca-Fernández, E. and Torres-Shoembert, R. (2020a). Valuation of the durability of the concrete used in the precast Great Soviet Panel System. Revista Facultad de Ingeniería 29(54), e10486

Socarrás, Y.C., González, 1., Alvarez, E., González, M.M. y Roca, E. (2020b). Evaluación de la calidad del hormigón en edificaciones construidas con el sistema prefabricado gran panel soviético. Tecnología Química 40(2), 264-277

Socarrás, Y.C., Alvarez, E. y Moreno, E. (2020c). Repercusiones de las contravenciones estructurales e incremento de peso en el Sistema Gran Panel Soviético en Santiago de Cuba. Revista de Obras Públicas 3623, 74-82
Socarrás, Y.C. (2020). Procedimiento para la evaluación de daños sísmicos potenciales en el sistema prefabricado Gran Panel Soviético. Tesis doctoral, Universidad de Oriente, Cuba Socarrás, Y., Álvarez, E. and Lora, F. (2021a). Forecasts on the seismic behavior of buildings constructed with the Great Soviet Panel. DYNA 88(216), 145-151

Socarrás, Y., Álvarez, E. and Lora, F. (2021b). Changes in the fundamental periods of buildings constructed with the Great Soviet Panel. ESTOA 10(19), 220-235 